\title{
Thermal formation of carbynes
}

\author{
Tobia M. Mazzolari ${ }^{1}$ and Nicola Manini ${ }^{1,2}$ \\ ${ }^{1}$ Dipartimento di Fisica, Università di Milano, Via Celoria 16, 20133 Milano, Italy \\ ${ }^{2}$ CNR-IOM Democritos National Simulation Center, Via Bonomea 265, 34136 \\ Trieste, Italy \\ E-mail: tobiamazzo@gmail.com, nicola.manini@mi.infm.it
}

\begin{abstract}
We simulate the formation of $s p$ carbon chains (carbynes) by thermal decomposition of $s p^{2}$ carbon heated by a hot discharge plasma, by means of tightbinding molecular dynamics. We obtain and analyze the total quantity of carbynes and their length distribution as a function of temperature and density.

PACS numbers: 81.07.-b
\end{abstract}

\section{Introduction}

The $s p$ form of carbon, carbyne, has proved more elusive than its $s p^{3}$ (diamond) and $s p^{2}$ (graphite/graphene) counterparts. Recently, also in view of the potential exciting applications [1, 2, 3, 4, 5, 6, 7, 8, $s p$ carbon chains (spCCs, or carbynes) are being produced in significant amount and investigated extensively. Besides synthetic routes [9, 10, 11, 12, 13, 14] leading to spCCs bonded to molecular ligands and various mechanical or electromagnetic methods [15, 16, 17, 18, 19] addressing the single spCC, carbyne has been formed in substantial amounts (together with other carbon clusters) via homogenous reactions within the hot plasma produced by an electric discharge at the surface of a graphite electrode [20, 21, 22, 23, 24, 25]. Despite their high reactivity, a significant fraction of these carbynes survive landing on a solid surface, where they are typically stabilized by grafting to other carbon clusters, mainly of $s p^{2}$ hybridization [26]. After deposition, carbynes are detected in the nanostructured film by various kinds of spectroscopies [25, 27, 28, 29].

In the present work we investigate the early formation stages of spCCs at the interface of a graphite electrode with a hot plasma. Specifically, we investigate the length distribution $P(m)$ of $\mathrm{C}_{m}$ chains, and its dependence on the temperature of the hot plasma and on the local density of the available carbon material. The time evolution of $P(m)$ and its temperature dependence can provide information useful for the tuning of the plasma properties, in view of an optimization of the formation of carbynes of a desired length. A previous simulation work [30] used a similar model to investigate the transformation of $s p$ structures into ordered structure, such as fullerenes and nanotubes 


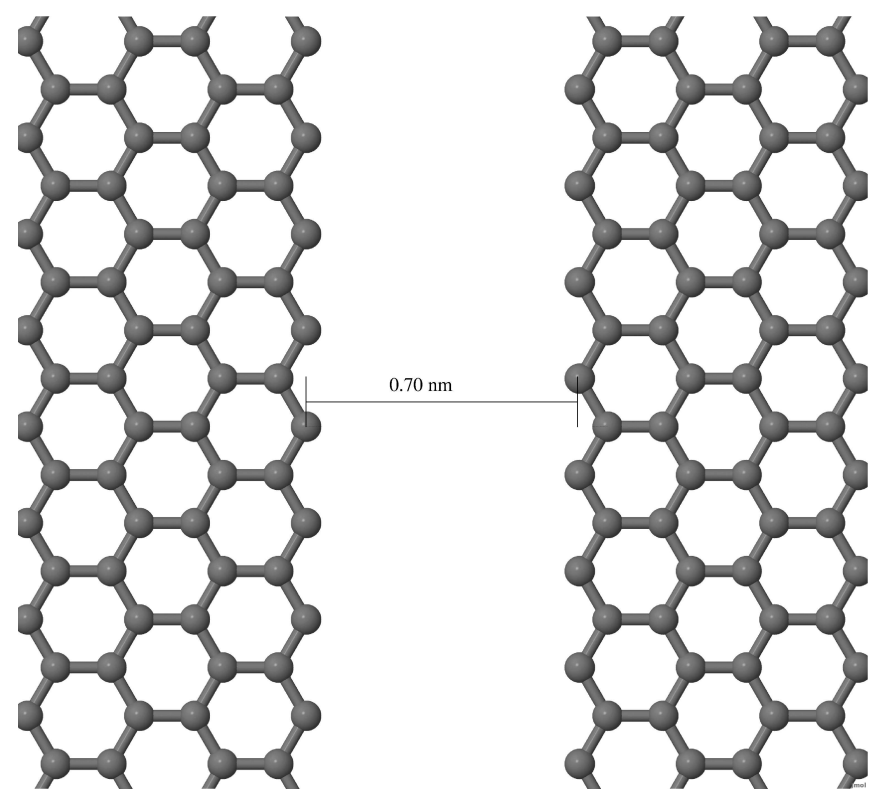

Figure 1: Starting configuration of the $T=0$ carbon ribbon, obtained by selecting a portion of an ideal graphene sheet. Periodic boundary conditions are applied over a parallelepiped box in all three orthogonal directions. The $\sim 0.70 \mathrm{~nm}$-wide central cut exposes the ribbon zig-zag edges.

under thermal annealing. Here we focus instead on a quantitative characterization of the initial formation of the carbynes, in a high-density, high-temperature plasma plume.

\section{Methods}

A reliable description of the $s p^{2} \rightarrow s p$ interconversion requires a transferable description of carbon binding in its different hybridizations. In view of these requirements, we adopt a well-established tight-binding (TB) model for the adiabatic potential of a carbon-only material [31]. In Appendix A we validate the adopted model against ab-initio density functional theory (DFT) simulations, in the local spin-density approximation (LSDA). As is standard in the literature of the field [30, 32, 33, 34], we evaluate the HellmannFeynman forces [35] for an efficient simulation of the classical time evolution of the ions of the carbon material.

We adopt a graphene nanoribbon to represent the initial state of the $s p^{2}$-carbon sacrificial electrode. A graphitic multilayer would make little difference because the adopted TB force field is rather short ranged and does not include Van-der-Waals interactions. The infinite nanoribbon is represented by a $s p^{2}$ carbon stripe inside a parallelepiped simulation cell, to which we apply periodic boundary conditions (PBC), see Fig. 1. We simulate the heat exchanges of the sample with the hot plasma and the bulk of the carbon electrode by means of a standard Langevin thermostat [36]. The 
Table 1: The general conditions used for the considered groups of simulations. The density is changed by varying the size of the simulation box in the direction perpendicular to the plane initially containing the nanonribbon.

\begin{tabular}{|c|c|c|c|c|c|c|}
\hline $\begin{array}{c}\eta \\
{\left[\mathrm{ps}^{-1}\right]}\end{array}$ & $\begin{array}{c}T \\
{[\mathrm{~K}]}\end{array}$ & $\begin{array}{l}\text { edge } \\
\text { type }\end{array}$ & $\begin{array}{l}\text { number } \\
\text { of atoms }\end{array}$ & $\begin{array}{c}\text { density } \\
{\left[\text { atoms nm }^{-3}\right]}\end{array}$ & $\begin{array}{l}\text { total time } \\
{[\mathrm{ps}]}\end{array}$ & $\begin{array}{l}\text { number of } \\
\text { simulations }\end{array}$ \\
\hline 0.1 & 4000 & zig-zag & 112 & 2.77 & 150 & 5 \\
\hline 0.1 & 4500 & zig-zag & 112 & 2.77 & 150 & 5 \\
\hline 0.1 & 5000 & zig-zag & 112 & 2.77 & 250 & 5 \\
\hline 0.1 & 6000 & zig-zag & 112 & 2.77 & 150 & 5 \\
\hline 1 & 4000 & zig-zag & 112 & 2.77 & 50 & 10 \\
\hline 1 & 4000 & zig-zag & 112 & 9.23 & 500 & 10 \\
\hline 1 & 4500 & zig-zag & 112 & 2.77 & 50 & 10 \\
\hline 1 & 4500 & zig-zag & 112 & 9.23 & 200 & 10 \\
\hline 1 & 5000 & zig-zag & 112 & 1.39 & 100 & 10 \\
\hline 1 & 5000 & zig-zag & 112 & 2.77 & 100 & 10 \\
\hline 1 & 5000 & zig-zag & 112 & 9.23 & 100 & 10 \\
\hline 1 & 5000 & zig-zag & 112 & 13.85 & 100 & 10 \\
\hline 1 & 5000 & zig-zag & 56 & 9.23 & 100 & 10 \\
\hline 1 & 5000 & armchair & 96 & 9.23 & 100 & 10 \\
\hline 1 & 6000 & zig-zag & 112 & 2.77 & 50 & 10 \\
\hline 1 & 6000 & zig-zag & 112 & 9.23 & 100 & 10 \\
\hline 5 & 4000 & zig-zag & 112 & 2.77 & 50 & 5 \\
\hline 5 & 4500 & zig-zag & 112 & 2.77 & 50 & 5 \\
\hline 5 & 5000 & zig-zag & 112 & 2.77 & 50 & 5 \\
\hline 5 & 6000 & zig-zag & 112 & 2.77 & 50 & 5 \\
\hline 10 & 4000 & zig-zag & 112 & 2.77 & 50 & 5 \\
\hline 10 & 4500 & zig-zag & 112 & 2.77 & 50 & 5 \\
\hline 10 & 5000 & zig-zag & 112 & 2.77 & 50 & 5 \\
\hline 10 & 6000 & zig-zag & 112 & 2.77 & 50 & 5 \\
\hline
\end{tabular}

equation of motion of each carbon atoms is

$$
\frac{d \mathbf{p}_{i}}{d t}=-\eta \mathbf{p}_{i}(t)+\mathbf{f}_{i}(t)+\tilde{\mathbf{f}}_{i}(t)
$$

where $\mathbf{p}_{i}$ is the momentum of atom $i, \mathbf{f}_{i}(t)$ is the (Hellmann-Feynman) force acting on atom $i$ due to the interaction with all other atoms in the sample, $\tilde{\mathbf{f}}_{i}(t)$ is a Gaussiandistributed stochastic force representing the collisions of atom $i$ with the hot plasma, and $\eta$ is the phenomenological parameter accounting for the rate of heat exchange with the thermostat [36]. We integrate these equations numerically with a $0.5 \mathrm{fs}$ time step. The other simulation details are summarized in Table 1 .

In the experiment, an electric discharge ionizes a short injected He-gas pulse. 

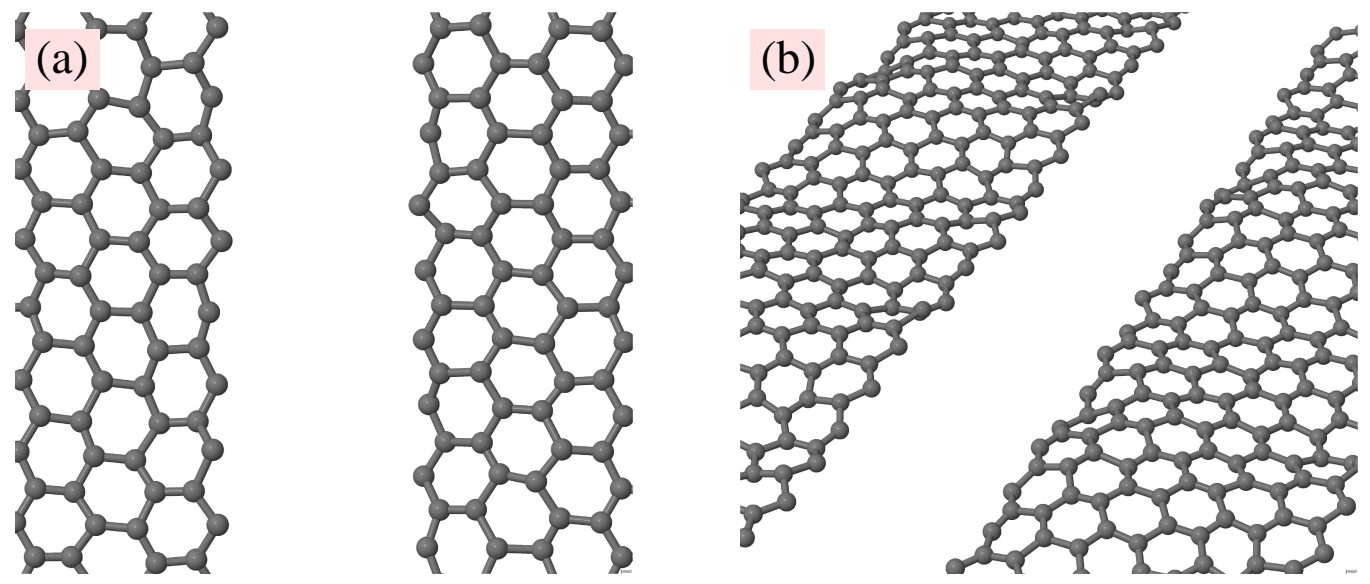

Figure 2: Initial configuration prepared starting from the ideal nanoribbon configuration of Fig. 1 1evolved for 4 ps at an intermediate temperature $T=2000 \mathrm{~K}$. (a) A top view of a single supercell; (b) an inclined perspective view of the infinitely repeated-cell geometry. When this snapshot is adopted as the starting point of successive simulations, the initial velocities are randomly reset according to a thermal distribution appropriate to the new simulation temperature. The PBC parallelepiped box remains unchanged, thus fixing the overall atomic density.

Ablation occurs when helium plasma strikes the carbon cathode surface, removing atoms via sputtering [20, 21, 22, 23]. The duration of the plasma pulse, in the submillisecond region [22], is orders of magnitude longer than typical sub-nanosecond times we can afford to explore in simulation. Over the nanosecond time scale, an essentially steady sputtering regime has established, with the progressive erosion of the bulk graphite electrode, and with the eroded carbon material diffusing away from the electrode through the hot plasma. Local thermal equilibrium is likely to be maintained by frequent collisions with the plasma, and successive progressive cooling is achieved over a time scale of microseconds, mainly by fragmentation and emission of electromagnetic radiation. In our atomistic model anything comparable to this steady decomposition regime is out of reach due to the huge number of atoms and far too long simulations times required. We rather gather information relevant to the experimental regime by investigating the transient decomposition of the nanoribbon when suddenly brought to high temperature.

Within the fixed simulation volume and at high temperature, the simulated sample progressively decomposes into isolated atoms, dimers, longer carbynes $\mathrm{C}_{m}$, and in principle also $s p^{2}$ fragments, although we observe few of the latter within the simulated conditions. In simulation, erosion proceeds until the $s p^{2}$ nanoribbon is completely decomposed and a final steady regime is reached, which can be described as a hot atomic/molecular carbon gas.

The dynamics of spCC formation can be addressed precisely by analyzing the transient regime where the nanoribbon gets eroded. To study this transient we adopt 


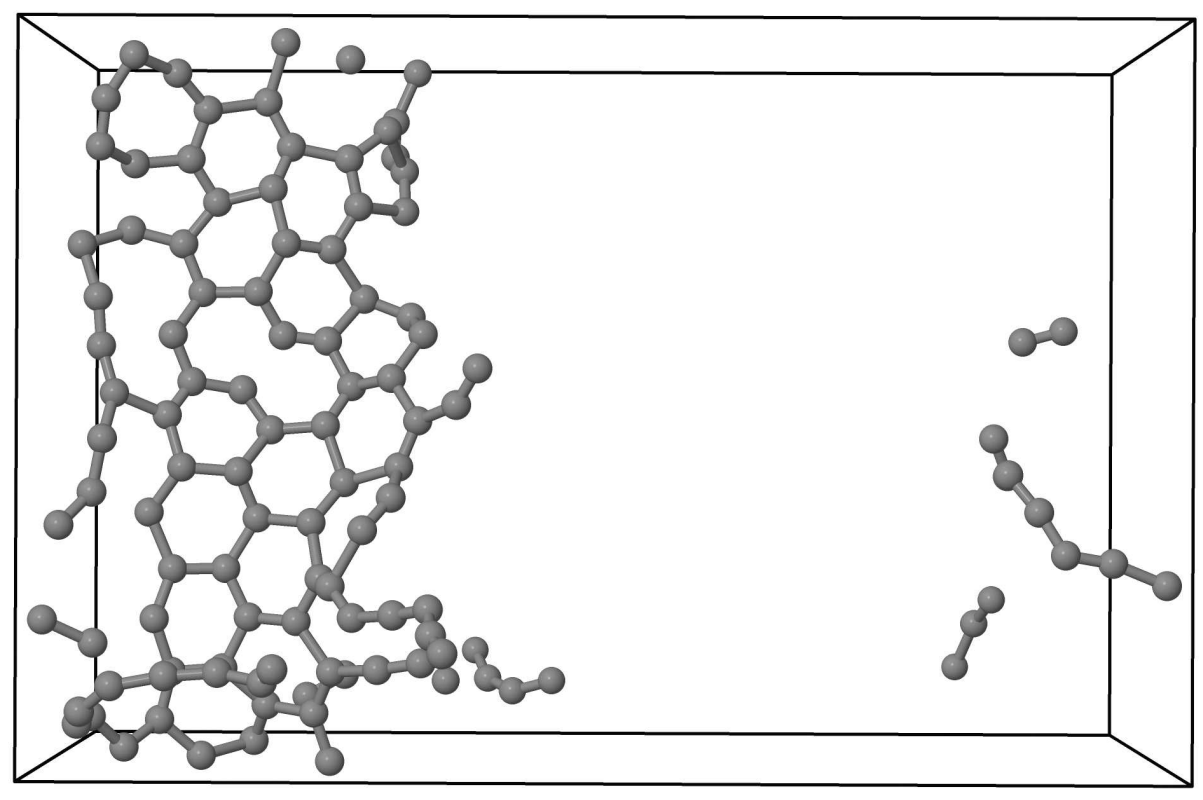

Figure 3: A typical snapshot of the $T=5000 \mathrm{~K}$ evolution of the sample starting off in the configuration of Fig. 2. Note an isolated $\mathrm{C}$ atom (top left), a dimer (mid-height right), a $\mathrm{C}_{3}$ (bottom right), a $\mathrm{C}_{4}$ (bottom center), a $\mathrm{C}_{8}$ chain crossing the box $\mathrm{PBC}$ and a few carbynes still partly attached to the $s p^{2}$ region.

the following protocol: (i) we start with an ideal $T=0$ configuration such as the one depicted in Fig. 1) (ii) we run a pre-thermalization simulation of 4 ps at the intermediate temperature $T=2000 \mathrm{~K}$, obtaining the configuration represented in Fig. 2 where a significant thermal excitation is already present in the phononic degrees of freedom, but no bonds are broken; (iii) we then attribute random initial velocities to the atoms, from a Maxwellian distribution at the desired simulation temperature, see Table 1, and start off the "production" Langevin run.

To generate a fair statistics, for given physical conditions we repeat the Langevin evolution at least 5 , but usually 10 times, starting from the same initial configuration but with independent random sets of initial velocities and stochastic forces $\tilde{\mathbf{f}}_{i}(t)$.

\section{Results}

The starting configuration is equilibrated, with both kinetic (velocities) and potential (positions) degrees of freedom representing the appropriate Boltzmann energy distribution at $T=2000 \mathrm{~K}$. As soon as the temperature is turned up to the "production" value listed in Table 1, the thermostat immediately transfers energy to the kinetic degrees of freedom. Subsequently, this extra energy is shared very rapidly with the potential degrees of freedom of the sample. This increased potential energy results in the progressive decomposition of the $s p^{2}$ sample, with the detachment of isolated atoms, clusters, and $s p$ chains of different lengths. We simulate temperatures which are 


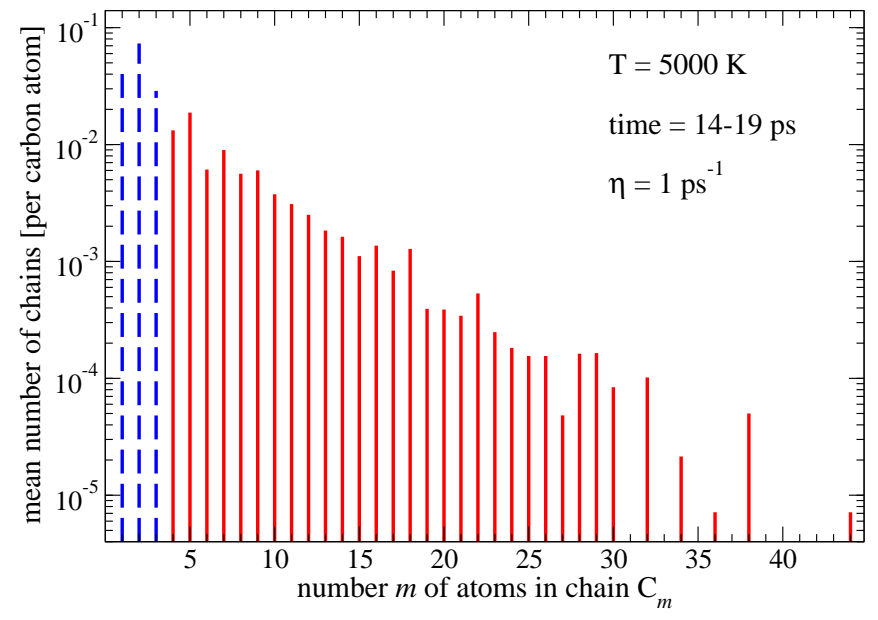

Figure 4: (Color online) Histogram of the frequency of individual spCC lengths. Data are averaged over 500 equally-spaced snapshots in the time interval from 14 to 19 ps of the evolution started when turning on a $T=5000 \mathrm{~K}$ Langevin thermostat characterized by a coupling rate $\eta=1 \mathrm{ps}^{-1}$. Further averaging is realized over 10 independent Langevin trajectories. The mean atomic density is $n=9.23 \mathrm{~nm}^{-3}$.

rather high compared to those which could be estimated in experiment, because they have the advantage to speed up the decomposition dynamics substantially, and bring it down to an accessible 10 ps time scale. At the considered temperatures and densities, isolated atoms and $s p$ chains are the dominant emitted clusters. As an example, Fig. 3 illustrates a typical snapshot where a $\mathrm{C}$ atom, $\mathrm{C}_{2}, \mathrm{C}_{3}, \mathrm{C}_{4}$ chains, and a $\mathrm{C}_{8}$ chain crossing the $\mathrm{PBC}$ are visible. All these carbynes are fully detached from the $s p^{2}$ nanoribbon. The main spCC formation mechanism involves random self-cleavage of the graphene edge, as visible e.g. at the left edge of the graphene sheet in Fig. 3 .

\subsection{The carbyne length distribution}

Figure 4 reports the distribution of length (expressed as number of atoms $m$ ) of the detaching $\mathrm{C}_{m}$ carbynes. This histogram includes isolated atoms and free chains and also carbynes under formation, still bound to the graphene edge at either or both ends. The $m=1$ column counts both isolated atoms and atoms attached with one bond to the graphene sheet. $s p^{2}\left(s p^{3}\right)$ atoms forming $3(4)$ bonds do not contribute to this statistics. To evaluate this histogram, we have developed a computer code which, taking PBC into account, identifies $s p$ atoms by being connected by either one or two bonds, and then follows the bonds and characterizes uniquely each carbyne.

The obtained distribution is dominated by single atoms, dimers and very short chains (dashed columns). For longer chains, i.e. proper carbynes, the length distribution decays approximately exponentially as $m$ increases. A small but detectable fraction extends to quite long carbynes with $m \geq 20$. A weak even-odd unbalance is visible.

The distribution depicted in Fig. 4 represents a snapshot of an evolving distribution, 


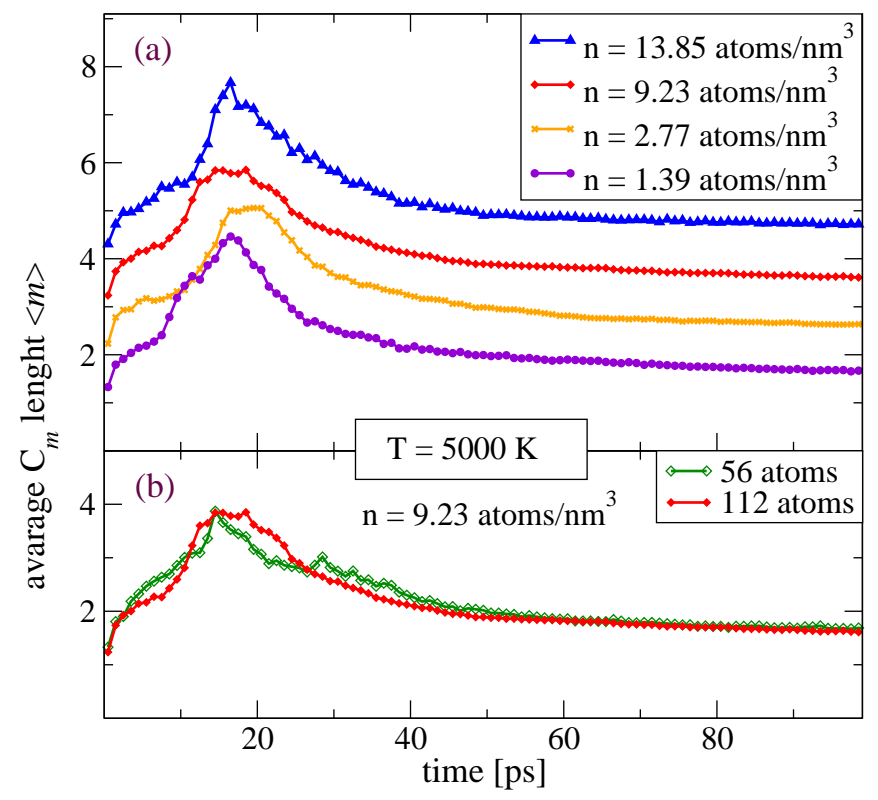

Figure 5: (Color online) The time evolution of the average length $\langle m\rangle$ of spCC from the simulation start at $T=5000 \mathrm{~K}$, averaged over 10 independent simulations and over 100 samples within the same ps. (a) Successive curves at different simulation-box volume, i.e. different mean atomic density, are mutually shifted upward by 1 for clarity. (b) Size effects illustrated by the comparison of the data for density 9.23 atoms $/ \mathrm{nm}^{3}$ with similar simulations (open symbols) carried out with a sample composed of one half of the atoms in one half volume.

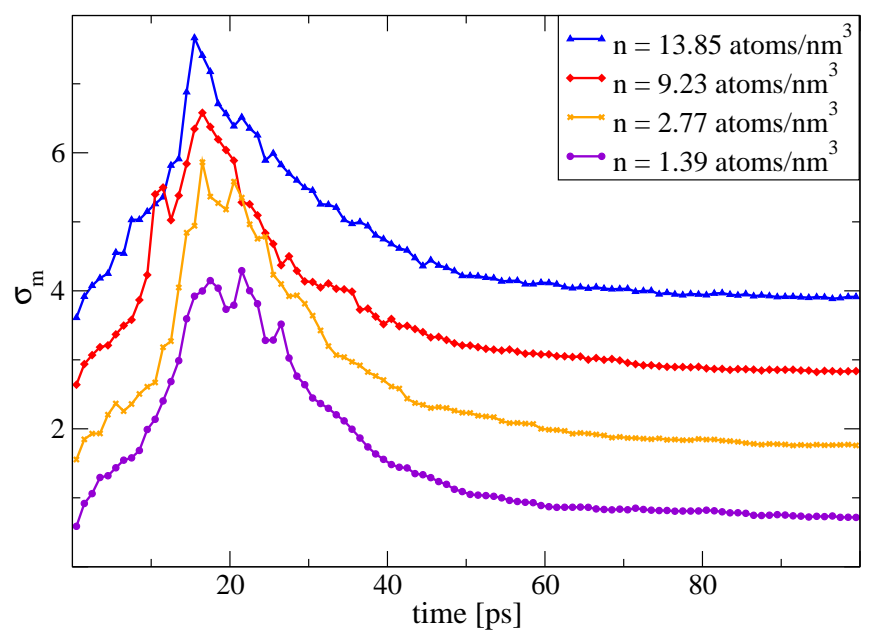

Figure 6: (Color online) The time evolution of the standard deviation of the spCC length distribution, as in Fig. 5. Successive curves at increasing mean atomic densities are shifted upward by 1 for readability. 


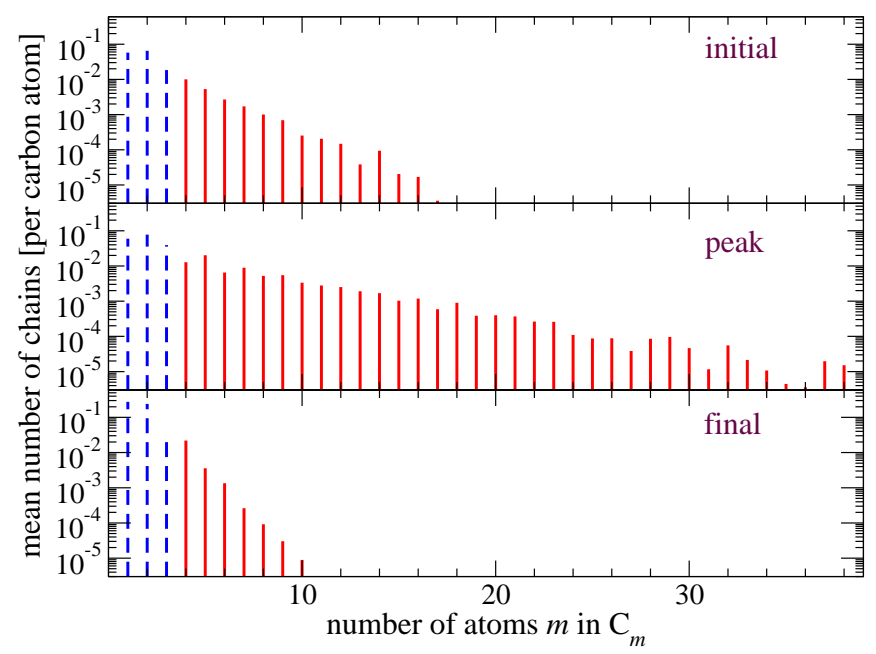

Figure 7: (Color online) Successive distributions of $\mathrm{C}_{m}$ lengths. The time intervals are: initial $-0 \div 10 \mathrm{ps}$; peak $-15 \div 25 \mathrm{ps}$; final $-90 \div 100$ ps. The simulation and statistical parameters are the same as in Fig. 4 .

and precisely as it occurs approximately 15 ps after turning on the interaction with the high-temperature environment. To characterize the time evolution of the chain-length distribution, Figs. 5(a) and 6 report respectively the average spCC length $\langle m\rangle$ and its fluctuation $\sigma_{m}=\left(\left\langle m^{2}\right\rangle-\langle m\rangle^{2}\right)^{1 / 2}$, as a function of time. The mean carbyne length $\langle m\rangle$ is seen to peak after approximately 20 ps of interaction with the hot thermostat, at a peak value $\langle m\rangle \simeq 4$. Successively, long spCCs decay rather rapidly, and the sample decays to mainly isolated atoms and dimers, with very few longer carbynes. The width $\sigma_{m}$ of the distribution, Fig. 6, follows a similar pattern, and is also especially broad (of the same order as $\langle m\rangle)$ in the 20 ps region.

We make sure that size effects are negligible by repeating similar calculations for a sample of one half the standard size, obtained by cutting the nanoribbon in Fig. 2 horizontally. The evolution of $\langle m\rangle$ shown in Fig. 5(b) shows little or no size effects. The detailed chain length distribution of the smaller-size simulation (not shown) is very similar to that of Fig. 4, but for a significant depletion of the long-chain region.

This evolution is illustrated also by the three histograms representing the initial, peak, and final time intervals, Fig. 7. We see that monomers and dimers dominate the distribution at all times, and especially in the long-time limit. Long carbynes are present as a significant fraction mainly in the $\langle m\rangle$-peak region, i.e. around 20 ps after starting to heat at $T=5000 \mathrm{~K}$. As we are interested in the formation of proper carbynes, from now on we focus on spCCs $\mathrm{C}_{m}$ with $m \geq 4$, namely those marked by solid lines in Figs. 4 and 7, with the understanding that these are a minority fraction of the entire emitted carbon material.

To investigate how the spCCs production depends on temperature, we run and compare multiple simulations at different temperatures. Figure 8 illustrates the evolution of the mean number $N_{m>3}$ of spCCs involving 4 atoms or more, for four 


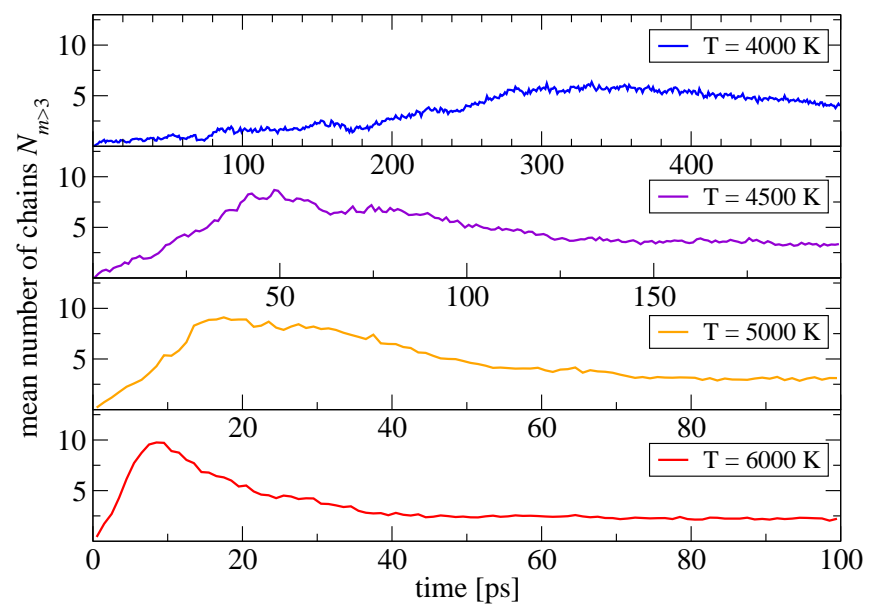

Figure 8: (Color online) The time evolution of the number of carbynes $\mathrm{C}_{m}$ with $m \geq 4$ atoms. Each curve at a different thermostat temperature is the result of averaging over 10 independent simulations and over 100 snapshots within each picosecond. The considered atomic density here is $n=9.23 \mathrm{~nm}^{-3}$.

different temperatures. The general trend of $N_{m>3}$ exhibits an initial increase, a broad peak, eventually followed by a systematic decay, regardless of temperature. Note however that the horizontal time scale is far more extended for the simulations carried out at lower temperature, where all decomposition phenomena occur over a far longer time scale. In the final region most $s p^{2}$ material has undergone a radical deterioration, and the sample has reached the state of an essentially gaseous mixture. The trend reported in Fig. 8 suggest that an optimal production rate of carbyne can be achieved provided that carbynes move out of the hot region in a time long enough for a significant concentration of carbynes to arise, but also short enough that the successive decomposition of these carbynes to dimers and isolated atoms has not proceeded significantly. This optimal time becomes shorter and shorter as the plasma temperature is raised.

\subsection{The intrinsic formation rate}

The formation rate of spCCs does not depend only on temperature but also on the effectiveness of the contact between the carbon sample and the hot plasma. In our model, we represent this contact by the strength $\eta$ of the coupling between the system and the thermostat. In experiment, this coupling is tuned by such properties as the local pressure, flux, and atomic mass of the hot plasma.

In addition to this "extrinsic" rate, we expect a "natural" contribution to the rate of formation of spCCs related to the intrinsic kinetic mechanisms involved in the erosion of the carbon material, and independent of the details of the coupling to the plasma/thermostat. To extract this "natural" formation rate of carbynes to $d N_{c} / d t \equiv d N_{m>3} / d t$, we need to subtract the thermostat contribution. To this purpose, 


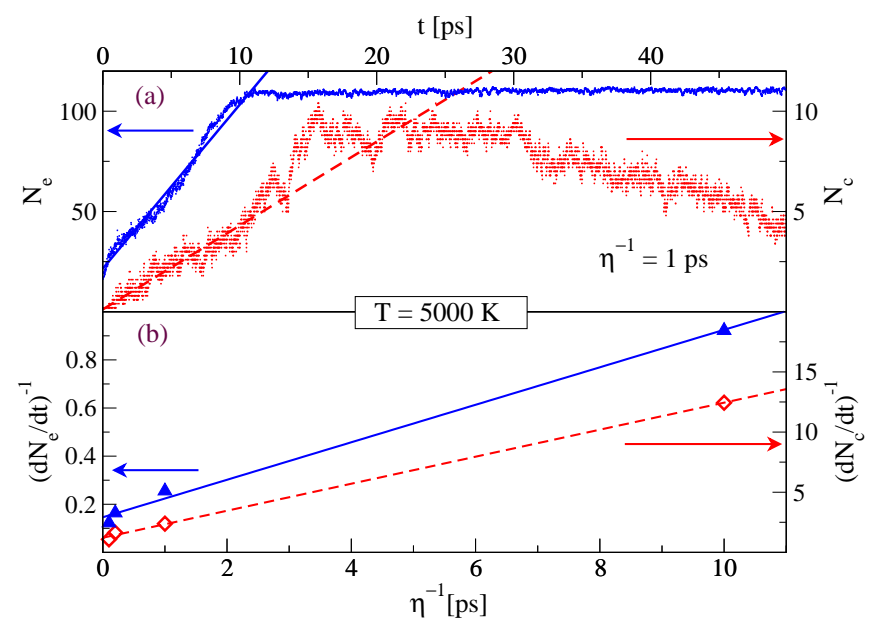

Figure 9: (Color online) The determination of the intrinsic generation rate for carbynes $\left(\tau_{c}\right)$ and for eroded carbon atoms $\left(\tau_{e}\right)$. (a) Firstly, the decomposition rate $d N_{e} / d t$ is determined as the slope of the linear fit of the initial raising time interval of a simulation. (b) Following Eq. (2) . the decomposition rates obtained for different thermostat coupling $\eta$ are plotted as a function of $\eta^{-1}$, and a further linear fit is carried out. The intercept resulting from these second fits provide precisely the best estimate of the intrinsic $\tau_{e}$ (and similarly $\tau_{c}$ ) for the considered simulation temperature $T$, as reported in Table 2 ,

Table 2: The intrinsic formation time for the generation of carbynes $\left(\tau_{c}\right)$ and for detached carbon atoms $\left(\tau_{e}\right)$ in any form (including carbynes). These data are obtained by a double fitting procedure illustrated in Fig. 9 .

\begin{tabular}{ccc}
\hline Temperature $[\mathrm{K}]$ & $\tau_{c}[\mathrm{ps}]$ & $\tau_{e}[\mathrm{ps}]$ \\
\hline 4500 & $4.22 \pm 0.31$ & $0.44 \pm 0.02$ \\
5000 & $1.22 \pm 0.14$ & $0.15 \pm 0.02$ \\
6000 & $0.19 \pm 0.04$ & $0.025 \pm 0.005$ \\
\hline
\end{tabular}

we simulate and compare the same conditions, with varied thermostat transfer rate $\eta$.

The detachment of one or multiple carbynes is an endothermic process requiring the concentration of energy at some point of the sample. This barrier energy $E_{B}$, in the $\mathrm{eV}$ range, is provided at the expense of the sample thermal energy, and it is eventually restored to the whole sample by the thermostat. This scheme configures the "series" of two processes occurring at definite rates: (i) the carbyne formation itself, at an intrinsic rate $\tau_{c}^{-1}$, and (ii) the transfer of heat from the thermostat, at a rate $\eta$. As these processes are not concurrent (in parallel) but sequential (in series, like chains of radioactive decays), the overall rate $d N_{c} / d t$ satisfies

$$
\left(\frac{d N_{c}}{d t}\right)^{-1}=D \eta^{-1}+\tau_{c},
$$

where $D$ is a dimensionless coefficient of order unity. $d N_{c} / d t$ is extracted by fitting the 


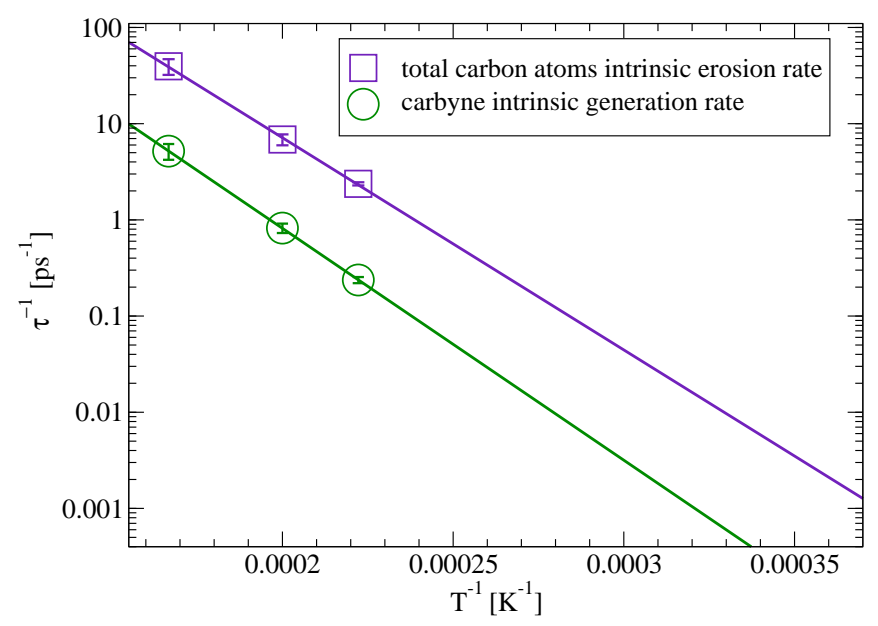

Figure 10: (Color online) The activated temperature dependence of the intrinsic generation rate of the chains $\tau^{-1}$. The data points are those reported in Table 2, the solid line is a best fit.

initial linearly-increasing region of the computed $N_{c}$ as a function of time, as in Fig. 8 , and specifically Fig. 9a. Given these values of $d N_{c} / d t$ computed at varied $T$ and $\eta$, we extract the intrinsic formation time $\tau$ for a spCC by fitting the observed $\left(d N_{c} / d t\right)^{-1}$ as a function of $\eta^{-1}$. The data are compatible with a straight line, see Fig. 9b. According to Eq. (2), the intercept at $\eta^{-1}=0$ provides precisely $\tau_{c}$. We repeat this procedure for different temperature, and obtain the intrinsic formation time $\tau_{c}$, listed in Table 2 .

Figure 10 reports the obtained rates $\tau_{c}^{-1}$ as a function of the inverse temperature. The variation of the intrinsic formation rate $\tau_{c}^{-1}$ is compatible with an Arrhenius law

$$
\log \left(\tau_{c}^{-1}\right)=\log \left(\tau_{c \infty}^{-1}\right)-\frac{E_{B}}{k_{\mathrm{B}} T} .
$$

The estimated barrier $E_{B}=4.78 \pm 0.02 \mathrm{eV}$, of course in agreement with the carbyne breakup energy of Fig. A1 in Appendix A. The attempt rate $\tau_{\infty}^{-1} \simeq 5.4 \times 10^{16} \mathrm{~s}^{-1}$ is quite large compared to typical attempt rates of molecular chemical reactions. The reason is that this rate refers to the entire sample.

An analogous analysis carried out for the total number $N_{e}$ of eroded carbon atoms (also illustrated in Figs. 9and 10) yields a similar barrier $(4.38 \mathrm{eV}$ ) and an approximately double attempt rate $\tau_{e \infty}^{-1} \simeq 1.8 \times 10^{17} \mathrm{~s}^{-1}$. We can assume that $\tau_{c}^{-1}$ and $\tau_{e}^{-1}$ are rates appropriate for the length of $s p^{2}$ carbon edges exposed to the plasma in our simulated sample (Fig. 2), i.e. approximately $4 \mathrm{~nm}$. Since the inter-layer distance in graphite is $0.335 \mathrm{~nm}$, and accounting from orientational disorder, our sample is equivalent to an exposed erodible surface area of approximately $S \simeq 1 \mathrm{~nm}^{2}$. At a plausible temperature next to the graphite surface, say $2500 \mathrm{~K}$, the extrapolated intrinsic rate $\tau_{e}^{-1} \simeq 3 \times 10^{8} \mathrm{~s}^{-1}$ is compatible with an erosion rate $\left(S \tau_{e}\right)^{-1} \simeq 3 \times 10^{26} \mathrm{~s}^{-1} \mathrm{~m}^{-2}$, corresponding to $\sim 5 \mathrm{~kg} \mathrm{~s}^{-1} \mathrm{~m}^{-2}$. This mass rate accounts for an erosion speed in the order of $\sim 2 \mathrm{~mm} / \mathrm{s}$. This figure is quite close to the electrode erosion speed observed during the active plasma-pulse part of the source cycle in experiment [20, 22. Of this eroded carbon, the 


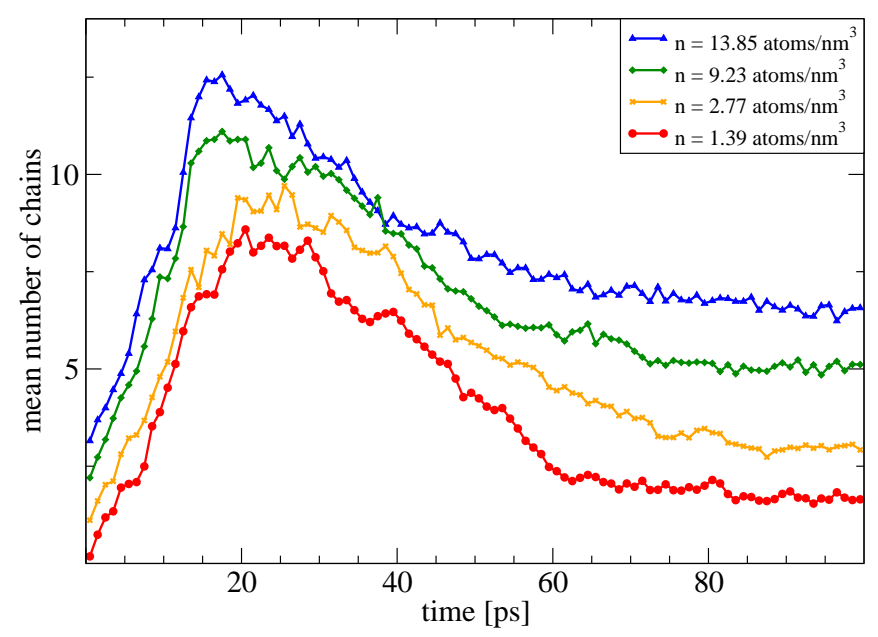

Figure 11: (Color online) The time evolution of the number of spCC longer than $m=3$ atoms. Each curve at a different atomic density is the result of averaging over 10 independent simulations at $T=5000 \mathrm{~K}$ and is shifted upward by 1 for readability.

fraction of "native" $m>3 \mathrm{C}_{m}$ carbynes is estimated to approximately $22 \%$ at the same temperature of $2500 \mathrm{~K}$ (assuming an average carbyne length of 5).

\subsection{Dependence on density and edge type}

It is necessary to examine how the amount and fraction of formed spCCs depends on the overall carbon density in the formation region. In the experimental conditions, the carbon density drops rapidly from that of bulk graphite inside the rod to a dilute-gas figure downstream in the carrier gas flux away from the surface. As it is currently impractical to determine the precise atomic density in the erosion region [20, 22, 25], we explore a range of densities. The data of Fig. 11 (see also Figs. 5 and 6) indicate that the carbon density does not affect dramatically the carbyne formation. Mostly, a higher density tends to increase slightly the early-time formation rate of carbynes, thus anticipating the time at which the number of formed carbynes is maximum.

Until now, all simulations represent solid carbon with the nanoribbon characterized by a zig-zag edge, as in Figs. 11 and 2, To make sure that the carbyne formation rate is not affected by this assumption, we simulate also a nanoribbon exposing an armchair edge. Figure 12 reports the comparison of the carbyne formation. While the armchair edge proves significantly more stable, with a slower initial rate of spCC formation, the observed difference is not especially large, approximately a factor two. We conclude that the explored zig-zag model is well suitable for estimating the rates, which it probably slightly overestimates compared to a random mix of zig-zag and armchair edges as in actual graphite. 


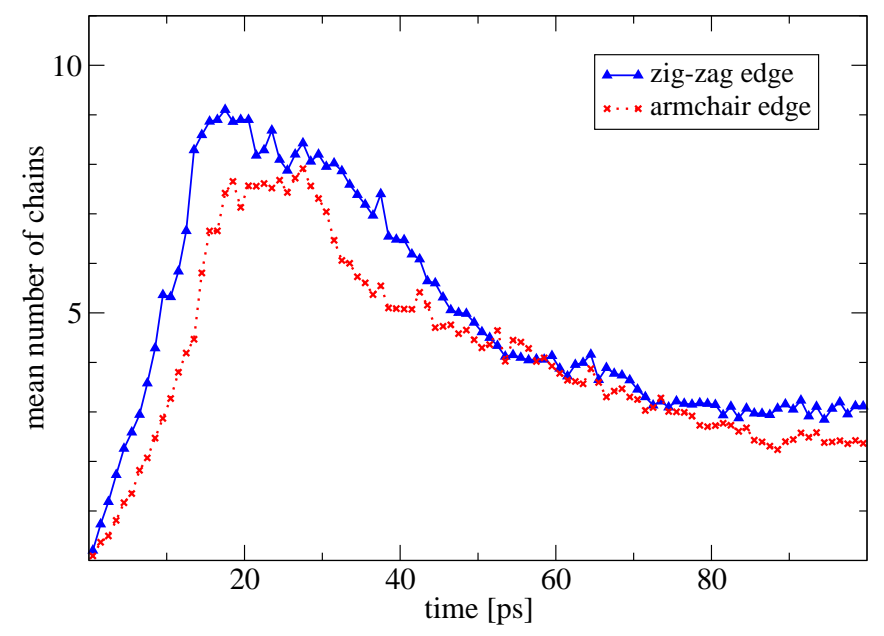

Figure 12: (Color online) Comparison of the number of spCC longer than $m=3$ atoms evolved from a $s p^{2}$ sheet terminated with armchair vs. zig-zag edges. Each curve is the result of averaging over 10 independent simulations at temperature $T=5000 \mathrm{~K}$ and the same atomic density $n=9.23 \mathrm{~nm}^{-3}$.

\section{Discussion and conclusions}

The adopted Langevin-thermostat model represents a substantial idealization of the erosion of $s p^{2}$ carbon interacting with a hot plasma. The significant inhomogeneity and temperature gradient occurring in the experimental setup are surely lacking in this model. All the same, the exploration of a range of temperature and densities shows that (i) temperature differences induce radical (activated) differences mainly in the overall production rate, but not on the long-time statistical properties of the formed carbynes; (ii) density variations induce minor changes on the carbyne formation. These results suggest that, despite its limitations, the adopted TB model allows us to explore the carbon decomposition and carbyne formation in a qualitative, but also statistically quantitative way.

In experiment, the carbynes and other produced clusters migrate into a cooler region where they may in part undergo fragmentation, recombination, and other transformations, including the synthesis of graphitic clusters, fullerenes, nanotubes, and amorphous structures. Contrary to the described carbyne formation, the recombination phenomena, already studied in the past [30], depend crucially on the probability of encounter and quenching of primary clusters, which in turn depends on the density of available carbon material. Accordingly, also the carbyne depletion between their generation in the hottest part of the plasma plume and the final deposition can occur at a rate strongly dependent on the plasma-gas pressure and flux. Further quantitative consideration of these phenomena may prove necessary in view of the direct interest in the formation of carbynes. 


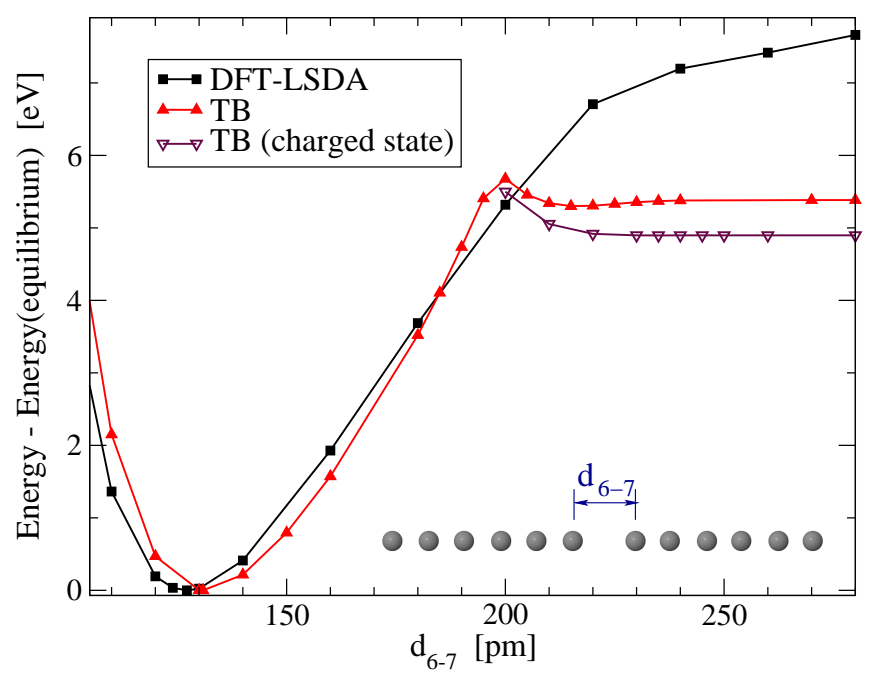

Figure A1: A comparison of the adiabatic potential energy of a $\mathrm{C}_{12} \mathrm{spCC}$ as a function of the length $d_{6-7}$ of the central bond, for a DFT-LSDA ab-initio model and the adopted TB model. In both calculations, all other bonds in the spCC are fully relaxed at each fixed elongation $d_{6-7}$ of the central bond. The TB calculation of the charged state (down triangles) yields a symmetry-broken configuration where two electrons localize on one $\mathrm{C}_{6}$ unit, thus effectively realizing a ion pair $\mathrm{C}_{6}^{2+}{ }_{-} \mathrm{C}_{6}^{2-}$.

\section{Acknowledgments}

We thank Nicola Ferri, Giovanni Onida, and Paolo Piseri for useful discussion.

\section{Appendix A. Validation of the TB potential}

The adopted parametric TB model [31] was constructed targeting mainly $s p^{3}$ and $s p^{2}$ carbon. Its adoption to describe spCCs should therefore be validated. To this purpose, we perform ab-initio DFT-LSDA simulations of a stretched carbyne. We adopt a planewaves basis (cutoff: 30 Rydberg) and ultrasoft pseudopotentials to account for core electrons, as implemented in the Quantum Espresso code [37. To test the reliability of the adiabatic potential resulting in the TB model, we compare the the TB total-energy adiabatic surface with the one obtained by DFT-LSDA for a $\mathrm{C}_{12}$ chain. In particular, we consider the variation of the adiabatic energy as the central bond between atoms 6 and 7 is stretched, with all other bonds fully relaxed. Figure A1 reports this comparison. The minimum energy is realized at a very similar bond length, within $4 \mathrm{pm}$, and the curvature in the minimum region is quite comparable. The main deviations occur when the central bond is stretched beyond $200 \mathrm{pm}$, approaching the cutoff distance (260 pm) of the TB model. The dangling bonds at the $\mathrm{C}_{12}$ ends produce a magnetic (spin-1) DFT ground state. The corresponding TB electronic structure exhibits a twofold-degenerate molecular orbital (half-)occupied by 2 electrons at the Fermi level. As the 6-7 bond is stretched, another level moves closer, until a further degeneracy is realized accounting 
for the two half-filled $\pi$ orbitals of the two identical $\mathrm{C}_{6}$ units. For $d_{6-7} \geq 200 \mathrm{pm}$, this degeneracy can be lifted by charge transfer of two electrons from one $\mathrm{C}_{6}$ fragment to the other, with a corresponding symmetry breaking and different bonding pattern of the two ions. This distortion leads to energy lowering, as illustrated in Fig. A1, This distortion however is an artifact of the TB model, which allows it because the TB model lacks any realistic description of the electrostatic charging energy. No sign of this artifact is observed in the DFT model, which instead switches from a total-spin $S=1$ to a $S=2$ state as $d_{6-7}$ exceeds $\simeq 220 \mathrm{pm}$.

Despite the risk of charging artifacts such as the one described above, the lack of magnetic exchange, and the short range, the overall shape of the adiabatic potential is essentially satisfactory to the purpose of the present calculations. The bondbreakage energy is approximately $5.5 \mathrm{eV}$, not too far from the DFT predicted value of approximately $8 \mathrm{eV}$. This underestimation of the binding energy, in turn, suggests

that the stability of carbynes and thus the tendency to form them in real life exceeds the one predicted by the present model simulations.

\section{References}

[1] B. Akdim and R. Pachter, ACS Nano 5, 1769 (2011).

[2] O. Cretu, A. R. Botello-Mendez, I. Janowska, C. P.-Huu, J.-C. Charlier, and F. Banhart, Nano Lett. 13, 3487 (2013).

[3] Y.-D. Guo, X.-H. Yan, and Y. Xiao, RSC Adv. 3, 16672 (2013).

[4] Z. Zanolli and J.-C. Charlier, Phys. Rev. B 81, 165406 (2010).

[5] Z. Zanolli, G. Onida, and J.-C. Charlier, ACS Nano 4, 5174 (2010).

[6] M. G. Zeng, L. Shen, Y. Q. Cai, Z. D. Sha, and Y. P. Feng, Appl. Phys. Lett. 96, 042104 (2010).

[7] E. Erdogan, I. Popov, C. G. Rocha, G. Cuniberti, S. Roche, and G. Seifert, Phys. Rev. B 83, 041401 (2011).

[8] M. Liu, V. I. Artyukhov, H. Lee, F. Xu, and B. I. Yakobson, ACS Nano 7, 10075 (2013).

[9] W. Mohr, J. Stahl, F. Hampel, and J. A. Gladysz, Chem. Eur. J. 9, 3324 (2003).

[10] X. Zhao, Y. Ando, Y. Liu, M. Jinno, and T. Suzuki, Phys. Rev. Lett. 90, 187401 (2003).

[11] Polyynes: Synthesis, Properties, and Applications, edited by F. Cataldo (CRC, Taylor\&Francis, London, 2005).

[12] W. A. Chalifoux and R. R. Tykwinski, C. R. Chimie 12, 341 (2009).

[13] C. A. Rice, V. Rudnev, R. Dietsche, and J. P. Maier, Astron. J. 140, 203 (2010).

[14] F. Cataldo, L. Ravagnan, E. Cinquanta, I. E. Castelli, N. Manini, G. Onida, and P. Milani, J. Phys. Chem. B 114, 14834 (2010).

[15] C. Jin, H. Lan, L. Peng, K. Suenaga, and S. Iijima, Phys. Rev. Lett. 102, 205501 (2009).

[16] A. Chuvilin, J. C. Meyer, G. Algara-Siller, and U. Kaiser, New J. Phys. 11, 083019 (2009).

[17] I. M. Mikhailovskij, E. V. Sadanov, T. I. Mazilova, V. A. Ksenofontov, and O. A. Velicodnaja, Phys. Rev. B 80, 165404 (2009).

[18] T. I. Mazilova, S. Kotrechko, E. V. Sadanov, V. A. Ksenofontov, and I. M. Mikhailovskij, Int. J. Nanoscience 9, 151 (2010).

[19] K. Inoue, R. Matsutani, T. Sanada, and K. Kojima, Carbon 48, 4209 (2010).

[20] E. Barborini, P. Piseri, and P. Milani, J. Phys. D: Appl. Phys. 32 L105 (1999).

[21] E. Barborini, P. Piseri, A. Li Bassi, A. C. Ferrari, C. E. Bottani, and P. Milani, Chem. Phys. Lett. 300, 633 (1999).

[22] P. Piseri, A. Podestà, E. Barborini, and P. Milani, Rev. Sci. Instr. 72, 2261 (2001). 
[23] L. Ravagnan, F. Siviero, C. Lenardi, P. Piseri, E. Barborini, and P. Milani, Phys. Rev. Lett. 89, 285506 (2002).

[24] C. S. Casari, A. Li Bassi, L. Ravagnan, F. Siviero, C. Lenardi, P. Piseri, G. Bongiorno, C. E. Bottani, and P. Milani, Phys. Rev. B 69, 075422 (2004).

[25] L. Ravagnan, P. Piseri, M. Bruzzi, S. Miglio, G. Bongiorno, A. Baserga, C. S. Casari, A. Li Bassi, C. Lenardi, Y. Yamaguchi, T. Wakabayashi, C. E. Bottani, and P. Milani, Phys. Rev. Lett. 98, 216103 (2007).

[26] I. E. Castelli, N. Ferri, G. Onida, and N. Manini, J. Phys.: Condens. Matter 24, 104019 (2012).

[27] L. Ravagnan, N. Manini, E. Cinquanta, G. Onida, D. Sangalli, C. Motta, M. Devetta, A. Bordoni, P. Piseri, and P. Milani, Phys. Rev. Lett. 102, 245502 (2009).

[28] E. Cinquanta, L. Ravagnan, I. E. Castelli, F. Cataldo, N. Manini, G. Onida, and P. Milani, J. Chem. Phys. 135, 194501 (2011).

[29] L. Ravagnan, T. Mazza, G. Bongiorno, M. Devetta, M. Amati, P. Milani, P. Piseri, M. Coreno, C. Lenardi, F. Evangelista and P. Rudolf, Chem. Commun. 47, 2952 (2011).

[30] Y. Yamaguchi, L. Colombo, P. Piseri, L. Ravagnan, and P. Milani, Phys. Rev. B 76, 134119 (2007).

[31] C. H. Xu, C. Z. Wang, C. T. Chan, and K. M. Ho, J. Phys.: Condens. Matter 4, 6047 (1992).

[32] F. Bonelli, N. Manini, E. Cadelano, and L. Colombo, Eur. Phys. J. B 70, 449 (2009).

[33] E. Cadelano, P. L. Palla, S. Giordano, and L. Colombo, Phys. Rev. Lett. 102, 235502 (2009).

[34] L. Ortolani, E. Cadelano, G. P. Veronese, C. Degli Esposti Boschi, E. Snoeck, L. Colombo, and V. Morandi, Nano Lett. 12, 5207 (2012).

[35] L. Colombo, Rivista Nuovo Cimento 28, 1 (2005).

[36] M. P. Allen and D. J. Tildesley, Computer Simulations of Liquids (Oxford University Press, Oxford, 1991).

[37] P. Giannozzi, S. Baroni, N. Bonini, M. Calandra, R. Car, C. Cavazzoni, D. Ceresoli, G. L. Chiarotti, M. Cococcioni, I. Dabo, A. Dal Corso, S. de Gironcoli, S. Fabris, G. Fratesi, R. Gebauer, U. Gerstmann, C. Gougoussis, A. Kokalj, M. Lazzeri, L. Martin-Samos, N. Marzari, F. Mauri, R. Mazzarello, S. Paolini, A. Pasquarello, L. Paulatto, C. Sbraccia, S. Scandolo, G. Sclauzero, A. P. Seitsonen, A. Smogunov, P. Umari, and R. M. Wentzcovitch, J. Phys.: Condens. Matter 21, $395502(2009)$. 